\title{
Distribution of highly repeated DNA sequences in Haynaldia villosa and its application in the identification of alien chromatin
}

\author{
ZHANG Wei ${ }^{1}$, ZHANG RuiQi $^{1}$, FENG YiGao ${ }^{1}$, BIE TongDe $^{1,2}$ \& CHEN PeiDu ${ }^{1 *}$ \\ ${ }^{1}$ National Key Laboratory of Crop Genetics and Germplasm Enhancement, Nanjing Agricultural University, Nanjing 210095, China; \\ ${ }^{2}$ National Wheat Improvement Subcenter, Yangzhou Academy of Agricultural Sciences, Yangzhou 225007, China
}

Received May 2, 2012; accepted October 26, 2012; published online January 9, 2013

\begin{abstract}
Haynaldia villosa (L.) is a wild relative species of common wheat that possesses many beneficial genes that can be used for wheat improvement. The accurate detection of $H$. villosa chromosomes in the genetic background of wheat is critical for transferring its beneficial genes to common wheat by chromosome engineering. The aim of the present study was to investigate the distribution patterns of two repeated DNA sequences, pSc119.2 and pAs1, as well as two rDNA multigene family sequences, 45S rDNA and 5S rDNA, in the individual chromosomes of $H$. villosa for the future precise identification of alien chromatin in germplasm development and breeding programs. A set of common wheat $H$. villosa disomic addition $1 \mathrm{~V}-7 \mathrm{~V}$ lines was used to determine these specific signals on individual chromosomes of $H$. villosa. The results showed that two rDNA probes, pTa71 (45S rDNA) and pTa794 (5S rDNA), were located on 1VS and 5VS, respectively, and the signal could be discriminated exclusively in the common wheat background as effective markers of 1VS and 5VS. Furthermore, all seven chromosomes of $H$. villosa could be distinguished clearly by fluorescence in situ hybridization using pSc119.2 and pAs1 as probes in combination. The utilization of these cytogenetic markers of repetitive sequences, combined with other molecular markers sometimes, will make it possible for a precise identification of alien chromosomes with high efficiency.
\end{abstract}

fluorescence in situ hybridization, Haynaldia villosa, repeated DNA sequence, wheat

Citation: Zhang W, Zhang R Q, Feng Y G, et al. Distribution of highly repeated DNA sequences in Haynaldia villosa and its application in the identification of alien chromatin. Chin Sci Bull, 2013, 58: 890-897, doi: 10.1007/s11434-012-5598-9

Wild relatives of common wheat (Triticum aestivum L., $2 n=$ $6 x=42$, AABBDD) are important sources of disease and pest resistance genes that can be employed in wheat improvement. Haynaldia villosa (L.) Schur (syn. Dasypyrum villosum (L.) Candargy) is an allogamous annual diploid relative $(2 n=2 x=14, \mathrm{VV})$ that is native to the northeastern part of the Mediterranean region. $H$. villosa possesses many important agronomical traits, such as disease resistance to powdery mildew, leaf and stem rusts, take-all, eyespot and wheat streak mosaic virus (WSMV) [1-5], as well as some elite genes that can increase the amount of seed storage protein [6], lysine content and gluten strength [7]. Therefore, $H$. villosa is an important resource for increasing genetic diversity in wheat improvement programs.

*Corresponding author (email: pdchen@njau.edu.cn)
The transfer of beneficial genes from $H$. villosa into common wheat was initiated in the 1970s at the Cytogenetics Institute, Nanjing Agricultural University, China. Since then, wheat- $H$. villosa hybrids, amphiploid, disomic addition lines $(1 \mathrm{~V}$ to $7 \mathrm{~V})$, substitution and translocation lines have been developed [2,3,8-10]. To better utilize the elite genes of $H$. villosa for wheat improvement through chromosome engineering, the development of approaches for the rapid and precise identification of $H$. villosa chromosomes or chromosome segments in wheat genetic background is particularly important in germplasm development and breeding processes.

Giemsa C- and N-banding have usually been used to detect alien chromosomes or chromosome segments in the wheat genetic background. Some researchers have distinguished individual $H$. villosa chromosomes by Giemsa C- 
and N-banding [11-13]. However, the distributed bands were not of a sufficient resolution for analysis and several chromosomes of $H$. villosa could not be distinguished accurately. The fluorescence in situ hybridization (FISH) technique, especially using highly repeated DNA sequences as probes, is an effective method for chromosome identification. With increasing numbers of commercially available fluorochromes, multi-color FISH offers the possibility of combining two or more DNA probes labeled with different fluorochromes in simultaneous and/or successive treatments on the same cells. The use of this technique has dramatically increased the resolution for the identification of all chromosomes within a species. In recent years, diverse repetitive sequences have been released with the development of genomics, and versatile probe combinations make it possible to establish a high resolution and unique karyotype of a species. Therefore, FISH-based chromosome identification methods are more powerful than the traditional chromosome banding techniques. The repetitive sequence $\mathrm{pSc} 119.2$ isolated from Secale cereale [14], the pAs1 isolated from Triticum tauschii [15], the ribosomal gene DNA sequence pTa71 (45S) and pTa794 (5S) [16] isolated from T. aestivum have been used to construct the chromosomal karyotype in common wheat and its relative species, while there has been a lack of investigation of their patterns in $\mathrm{H}$. villosa chromosomes.

The objective of this study was to construct a molecular karyotype of $H$. villosa chromosomes using pSc119.2, pAs1, 45S rDNA and 5S rDNA as FISH probes for the identification of individual $H$. villosa chromosomes or chromosome segments in a wheat genetic background, which would be helpful for the utilization of beneficial genes of $H$. villosa in future wheat improvement programs.

\section{Materials and methods}

\subsection{Plant materials}

The H. villosa parental line (91C43), originally introduced from the Cambridge Botanical Gardens, UK, was used as the donor parent in the production of wheat- $H$. villosa alien chromosome addition lines. The T. aestivum cultivar, Chinese Spring (CS), Triticum durum, T. durum-H. villosa amphiploid $(2 n=42$, AABBVV) and a set of T. aestivum- $H$. villosa disomic addition lines (AD $1 \mathrm{~V}-7 \mathrm{~V})$ were all maintained at the Cytogenetics Institute, Nanjing Agricultural University (CINAU), China.

\subsection{DNA probes and labeling}

The repeated DNA sequences, pSc119.2, pAs1, pTa71 (45S rDNA) and pTa794 (5S rDNA) were used for the FISH analyses. The probes were labeled with digoxigenin-11dUTP (Roche Diagnostics GmbH, Germany) or biotin-16dUTP (Roche Diagnostics GmbH, Germany) by the nicktranslation according to the manufacturer's protocols.

\subsection{Cytogenetic analyses}

The chromosome preparations of root-tip cells (RTCs) were made according to the method described by Gill et al. [17]. Genomic in situ hybridization (GISH) and fluorescence in situ hybridization (FISH) were performed as described by Zhang et al. [18] with minor modifications. The biotinylated probe was detected with FITC-conjugated avidin (Roche Diagnostics $\mathrm{GmbH}$ ) and digoxigenin-dUTP was detected with anti-digoxigenin rhodamine conjugate (Roche Diagnostics $\mathrm{GmbH}$ ). The slides were counterstained with 0.6 $\mathrm{ng} / \mu \mathrm{L}$ of propidium idodide (PI) for GISH and $2.5 \mathrm{ng} / \mu \mathrm{L}$ of 4',6-diamidino-2-phenylindole (DAPI) for FISH, and were mounted with Vectashield (Vector Laboratories, USA). Signals were then visualized using an Olympus BX60 fluorescence microscope (Olympus Corporation, Japan) with appropriate filters.

\subsection{Molecular markers analysis}

Two 3V-specific molecular markers and two 7V-specific molecular markers were used to identify the alien chromatin. Primer information is shown in Table 1. Genomic DNA was extracted using the SDS method described by Sharp et al. [19]. PCR amplifications were conducted in a $10 \mu \mathrm{L}$ reaction mixture containing $1 \times$ Taq DNA polymerase buffer, 0.8 $\mathrm{mmol} / \mathrm{L} \mathrm{MgCl}_{2}, 0.8 \mathrm{mmol} / \mathrm{L}$ dNTPs, $200 \mu \mathrm{mol} / \mathrm{L}$ primers, 2 U DNA polymerase and $50 \mathrm{ng}$ genomic DNA as the template. The samples were denaturated at $94^{\circ} \mathrm{C}$ for $3 \mathrm{~min}$ and subjected to 34 cycles of $30 \mathrm{~s}$ of denaturation at $94^{\circ} \mathrm{C}, 50 \mathrm{~s}$ annealing at $T_{\mathrm{m}}$ and $1.2 \mathrm{~min}$ elongation at $72^{\circ} \mathrm{C}$. A final

Table 1 Specific molecular markers used for the identification of chromosomes $3 \mathrm{~V}$ and $7 \mathrm{~V}$ of $H$. villosa

\begin{tabular}{|c|c|c|c|c|}
\hline Marker & Type & EST ID & Chromosome arm & Primer sequence $\left(5^{\prime} \rightarrow 3^{\prime}\right)$ \\
\hline $\mathrm{X}_{\text {CINAU38-250 }}$ & EST-STS & BJ216564 & $3 \mathrm{VS}$ & $\begin{array}{l}\text { Forward: CGACGACGTAGATCCAGATG } \\
\text { Reverse: TGCTCATGATCGTCATCTCC }\end{array}$ \\
\hline $\mathrm{X}_{\mathrm{HVM} 64-200}$ & Barely-SSR & & $3 \mathrm{VL}$ & $\begin{array}{l}\text { Forward: GATGTGAAGGCTGCCCTG } \\
\text { Reverse: ACACGCCCTATTACCCAGTG }\end{array}$ \\
\hline $\mathrm{X}_{\text {CINAU256-800 }}$ & EST-STS & BE446380 & $7 \mathrm{VS}$ & $\begin{array}{l}\text { Forward: TCACCTCCCTACCTGTCA } \\
\text { Reverse: ACGAAATGCTTGGTCCTT }\end{array}$ \\
\hline $\mathrm{X}_{\text {CINAU261-1300 }}$ & EST-STS & & $7 \mathrm{VL}$ & $\begin{array}{l}\text { Forward: GGTTTGGACTGAATGGTGCT } \\
\text { Reverse: TGCATTTGCCTTGCTGATAG }\end{array}$ \\
\hline
\end{tabular}


cycle with an extension of $10 \mathrm{~min}$ at $72^{\circ} \mathrm{C}$ was applied to complete the reactions. The PCR products were analyzed on $8 \%$ polyacrylamide gels in $1 \times$ TBE buffer.

\section{Results}

\subsection{Distribution of highly repeated DNA sequences in H. villosa chromosomes}

The repeated DNA sequences were used as FISH probes to identify individual $H$. villosa chromosomes. Figure 1(a), (c) and (d) shows the somatic metaphase chromosomes of $H$. villosa after FISH with pSc119.2, 45S rDNA and 5S rDNA as probes, respectively, and after counterstaining with 4', 6-diamidino-2-phenylindole (DAPI). The pSc119.2 as a probe for FISH has a relatively simple distribution among each pair of chromosomes, its signals are mainly located on the terminal or sub-terminal sites of both arms in five pairs of chromosomes, but are located on the short arms in the other two pairs of chromosomes (Figure 1(a)). The signals generated by the $\mathrm{pSc} 119.2$ probe differed in position and their signal intensity could be identified uniquely. The hybridization sites of the $45 \mathrm{~S}$ rDNA are located on the short arm of chromosome $1 \mathrm{~V}$, which is a satellite (SAT) chromosome (Figure 1(b) and (c)), while the hybridization sites of 5S rDNA are located on the short arm of chromosome $5 \mathrm{~V}$, whose arm ratio is the biggest in all seven chromosomes of $H$. villosa (Figure $1(\mathrm{~d})$ ). These results were in ac- cordance with the findings of Yuan et al. [20] in that only chromosome 1VS and 5VS could be identified using the $45 \mathrm{~S}$ rDNA probe and $5 \mathrm{~S}$ rDNA probe, respectively. It was found that pAs1 hybridization signals were distributed on all the chromosome arms of $H$. villosa (Figure 1(e)), including signals at the terminal, sub-terminal or interstitial sites and, occasionally, at centromeric positions. Furthermore, when pSc119.2 and pAs1 were used as probes to hybridize with the $H$. villosa chromosomes in dual-color FISH, the pAs1 signals were more widely dispersed than those of pSc119.2 (Figure 1(f)).

\subsection{Assignment of the FISH signals to specific chromo- somes using a set of wheat- $H$. villosa disomic addition lines}

A set of $T$. aestivum- $H$. villosa disomic addition lines, which were confirmed by molecular marker analysis (Table $\mathrm{S} 1$ and Figure S1), were used to assign the FISH signals distributed on individual chromosomes of $H$. villosa through sequential GISH (Figure 2(a)-(g), left) and dual-color FISH using pSc119.2 (green) and 45S rDNA (red) as probes (Figure 2(a)-(g), right). A pair of $H$. villosa chromosomes in each disomic addition lines appeared as a unique FISH pattern, and therefore chromosomes 1V-7V could be distinguished. An idiogram of $H$. villosa chromosomes was made as shown in Figure 3. Using pSc119.2 and pAs1 as probes in combination, all of the chromosome arms of $H$. villosa could be distinguished clearly. For example,

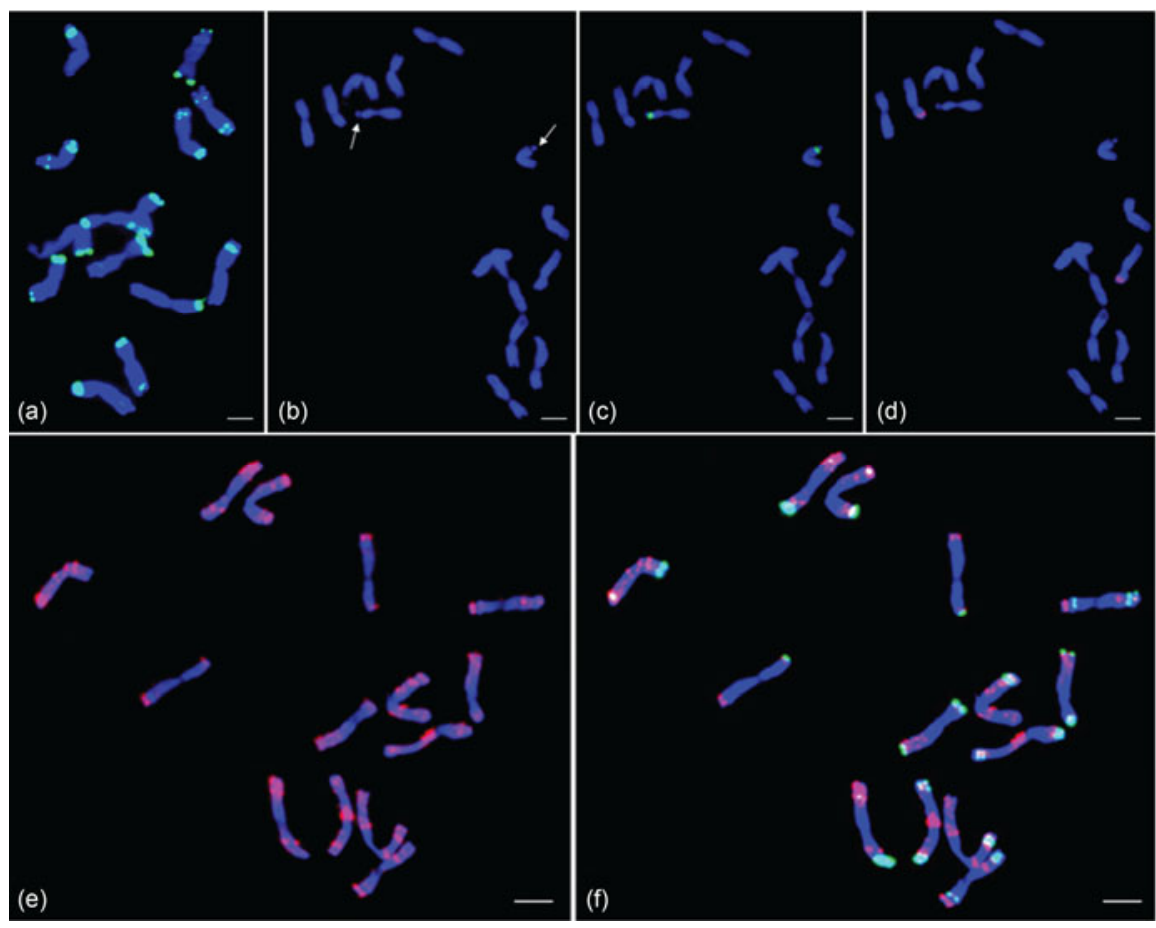

Figure 1 Fluorescence in situ hybridization analysis of chromosome in H. villosa. (a) Biotin-labeled pSc119.2 (green), (b) counterstained with DAPI (the SAT chromosomes are arrowed), (c) biotin-labeled 45S rDNA (green), (d) digoxigenin-labeled 5S rDNA (red), (e) digoxigenin-labeled pAs1 (red), (f) dualcolor FISH with pSc119.2 (green) and pAs1 (red). Scale bars, $10 \mu \mathrm{m}$. 

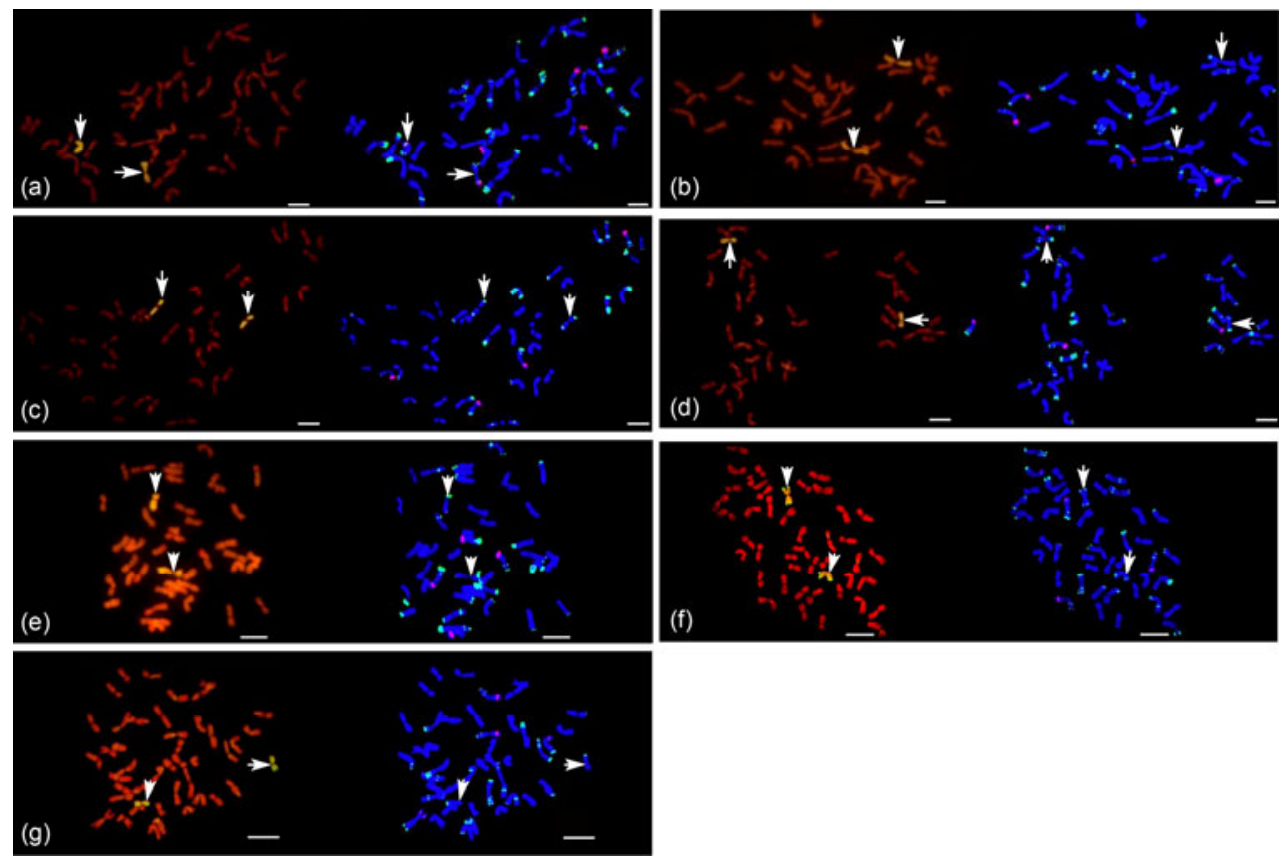

Figure 2 Chromosome sequential genomic in situ hybridization (left) and fluorescence in situ hybridization (right) on root tip cells of a set of wheat- $H$. villos a disomic addition lines, arrows show one pair of $H$. villosa chromosomes in each figure, simultaneous visualization of hybridization sites to pSc119.2 (green) and 45S rDNA (red) in FISH, signals of 45S rDNA on wheat chromosomes are $1 \mathrm{~B}$ and $6 \mathrm{~B}$ in each figure. (a)-(g) shows $1 \mathrm{~V}, 2 \mathrm{~V}, 3 \mathrm{~V}, 4 \mathrm{~V}, 5 \mathrm{~V}, 6 \mathrm{~V}$ and $7 \mathrm{~V}$ addition lines, respectively. Scale bars, $10 \mu \mathrm{m}$.

the signals of pSc119.2 only appeared on the short arm of $2 \mathrm{~V}$ and $7 \mathrm{~V}$, but the signals of pAs 1 on $2 \mathrm{~V}$ were more widely dispersed than those of pAs1 on 7V. Consequently, both the chromosome arms of $2 \mathrm{~V}$ and $7 \mathrm{~V}$ could be distinguished by utilizing pSc119.2 and pAs1 as probes in combination (Figure 3).

\subsection{The application of FISH landmarks in the identifi- cation of alien chromatin}

The pollen of $T$. durum- $H$. villosa amphiploid was irradiated with ${ }^{60} \mathrm{Co} \gamma$-rays $[21,22]$ in order to develop more wheat- $H$.

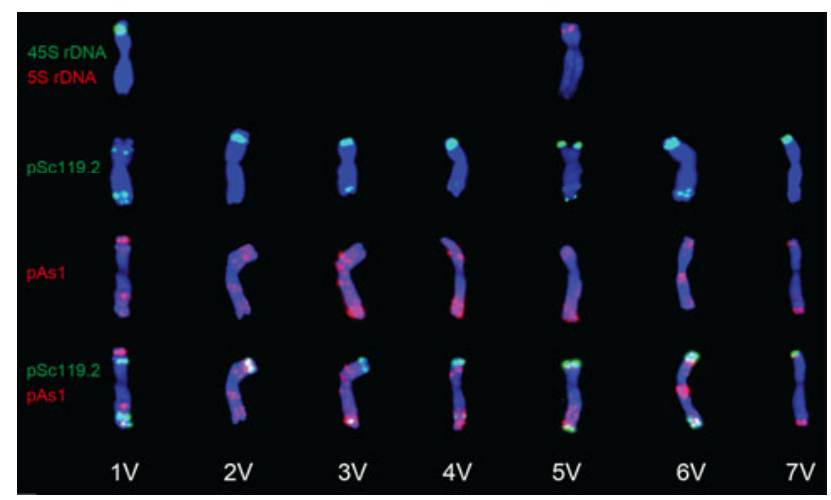

Figure 3 Idiogram of the chromosomes of $H$. villosa showing locations of $45 \mathrm{~S}$ rDNA (green), 5S rDNA (red), pSc119.2 (green), pAs1 (red) and simultaneous visualization of pSc119.2 (green) and pAs1 (red). The chromosome nomenclature was based on their homology to wheat and $\mathrm{H}$. villosa chromosomes. villosa translocations that are involved in different chromosomes and chromosome segments of $H$. villosa. We screened numerous $H$. villosa chromosome structural changes in the offspring that included whole-arm translocations, small alien segment translocations, large alien segment translocations and the terminal deletion of $H$. villosa chromosomes by GISH. However, some wheat- $H$. villosa translocations, especially small alien segment translocations, could not be identified accurately with the C-banding method. Therefore, some chromosome structural changes were used to test the usefulness of these FISH landmarks for the identification of chromosome segments of $H$. villosa.

As 45S rDNA and 5S rDNA were marked for 1VS and $5 \mathrm{VS}$, respectively, the chromosome structural changes of $H$. villosa were initially analyzed using $45 \mathrm{~S}$ rDNA and $5 \mathrm{~S}$ rDNA as probes. These results showed that three chromosome structural changes (TV277-7, TV288-4 and TV54-4-1) that involved 1VS and four (TV49-1-6, TV08-3, TV179-3-3 and TV330-6) that involved 5VS (Figure 4) could be easily identified. Subsequently, the seven chromosome structural changes were confirmed to be chromosome specific molecular markers of $1 \mathrm{~V}$ and 5V (Tables S2 and S3), and the results were consistent with those of sequential GISH/FISH assays.

The second example for the successful use of the established karyotype to determine the identity of chromosomes was to characterize two alien telocentric chromosomes in two plants, TV60-1-8-18 and TV60-1-8-27, identified in the offspring of irradiated $T$. durum-H. villosa amphiploid. To characterize the two telocentric chromosomes, the somatic 


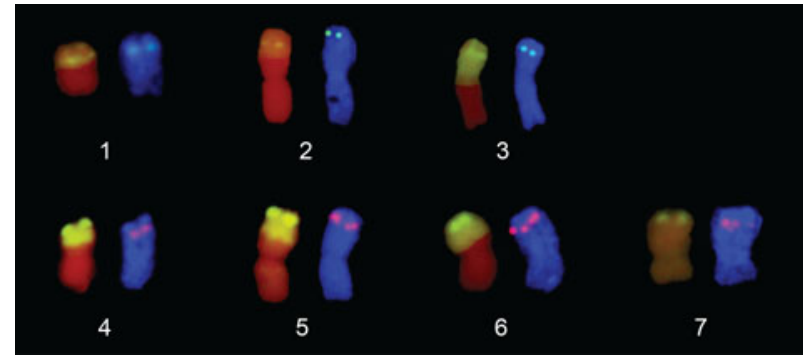

Figure 4 Chromosome sequential genomic in situ hybridization and fluorescence in situ hybridization of $H$. villosa chromosome structural changes involving 1VS (1-3) and 5VS (4-7). Total genomic DNA of $H$. villosa labeled with fluoresein-12-dUTP (green, GISH, left), 45S rNDA labeled with biotin-16-dUTP (green, FISH, right) and 5S rDNA labeled with digoxigenin-11-dUTP (red, FISH, right). 1, TV277-7; 2, TV288-4; 3, TV54-4-1; 4, TV49-1-6; 5, TV08-3; 6, TV179-3-3; 7, TV330-6.

chromosomes at metaphase were hybridized with biotin-labeled pSc119.2 and digoxigenin-labeled pAs1 (Figure $5)$. The result of the comparison of FISH-patterns showed that a telocentric chromosome in TV60-1-8-27 plants was a short arm of 3V (Figures 5(a) and 6) and a telocentric chromosome in TV60-1-8-18 plants was a long arm of 3V (Figures 5(b) and 6). Furthermore, the two telosomic plants were analyzed with the chromosome arm-specific molecular markers of $3 \mathrm{VS}$ and $3 \mathrm{VL}, \mathrm{X}_{\mathrm{CINAU} 38-250}$ and $\mathrm{X}_{\mathrm{HVM} \text { 64-200 }}$ (Figure 7), and the results were consistent with those of sequential GISH/FISH assays.

In addition, two whole-arm translocation plants were identified by in situ hybridization using biotin-labeled pSc119.2 and digoxigenin-labeled pAs1 as probes (Figure $8)$. The result of the comparison of FISH-patterns showed
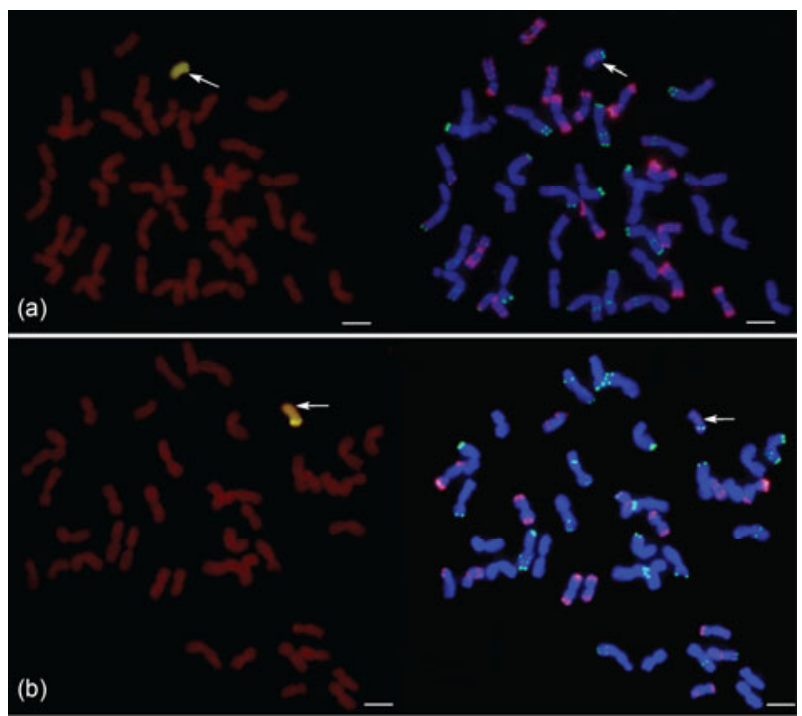

Figure 5 Chromosome sequential GISH (left) and FISH (right) on root tip cells of lines TV60-1-8-27 and TV60-1-8-18, where the pSc119.2 signal is green and pAs1 signal is red, and arrows show a telocentric chromosome. (a) The TV60-1-8-27 plant is a mono-telosomic addition line MAt.3VS, while (b) the TV60-1-8-18 plant is a mono-telosomic addition line MAt.3VL. Scale bars, $10 \mu \mathrm{m}$.

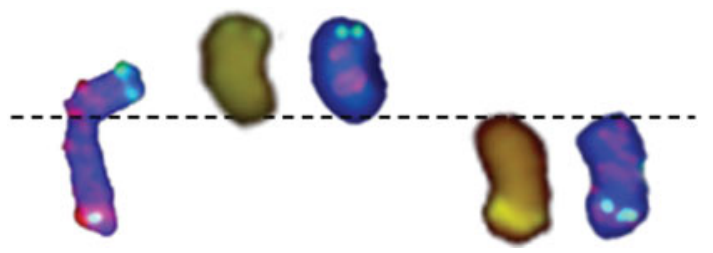

Figure 6 The pattern of dual-color FISH of $3 \mathrm{~V}$ chromosome and two telocentric chromosomes, from left to right, chromosome $3 \mathrm{~V}$, short arm of $3 \mathrm{~V}$ telocentric chromosome (in TV60-1-8-27) and long arm of $3 \mathrm{~V}$ telocentric chromosome (in TV60-1-8-18), the pSc119.2 is green and pAs1 is red.

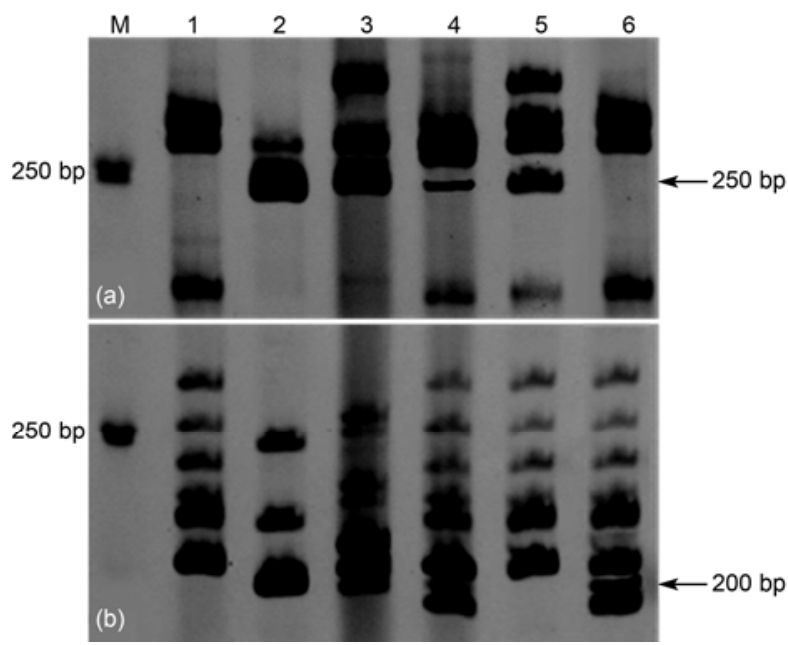

Figure 7 An example of PCR analysis of the two telocentric chromosomes using marker $\mathrm{X}_{\mathrm{CINAU} 38-250}(3 \mathrm{VS})$ and $\mathrm{X}_{\mathrm{HVM} 64-200}$ (3VL). (a) Amplification of primer CINAU38, (b) amplification of primer HVM64, arrows show a specific band for chromosome 3V. M, DNA Marker DL2000; 1, Chinese Spring; 2, H. Villosa; 3, T. durum-H. villosa amphiploid; 4, DA3V; 5, TV60-1-8-27; 6, TV60-1-8-18.

that the translocation chromosome in the TV54-5-5-22 plant involved 7VS (Figures 8(a) and 9), while the translocation chromosome in TV54-5-5-21 plant involved 7VL (Figures 8(b) and 9). Furthermore, the two whole-arm translocations were analyzed with chromosome arm-specific molecular markers of 7V, $\mathrm{X}_{\mathrm{CINAU} 256-800}(7 \mathrm{VS})$ and $\mathrm{X}_{\mathrm{CINAU} 261-1300}(7 \mathrm{VL})$ (Figure 10), and the results were consistent with those of sequential GISH/FISH assays.

\section{Discussion}

Plant genomes usually consist of a high percentage of repetitive sequences, which are generally dispersed along the plant chromosomes [23]. However, some special repetitive families are only distributed in particular chromosome regions [24]. The distinct sequence composition and distribution of these repetitive families may be due to duplications, diversifications, homogenization and deletions. Therefore, the characterization of the repetitive families makes it possible to distinguish different genomes and chromosomes according to their unique distribution patterns or sequence poly 

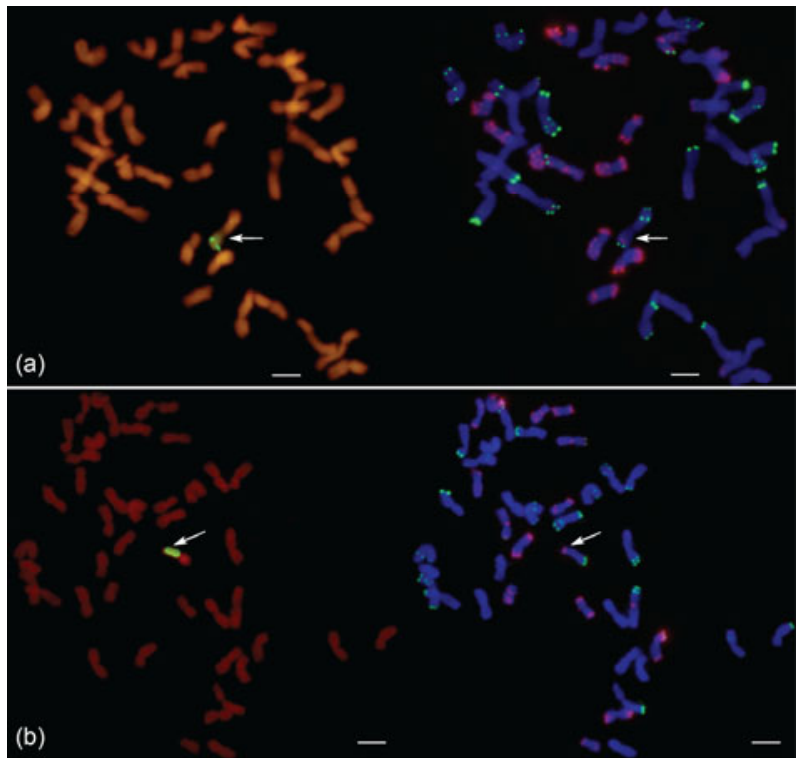

Figure 8 Chromosome sequential GISH (left) and FISH (right) on root tip cells of lines TV54-5-5-22 and TV54-5-5-21, the pSc119.2 signal is green and pAs1 signal is red, arrows show a translocation chromosome. (a) The TV54-5-5-22 plant with a translocation chromosome involved in 7VS, while (b) the TV54-5-5-21 plant with a translocation chromosome involved in 7VL. Scale bars, $10 \mu \mathrm{m}$.

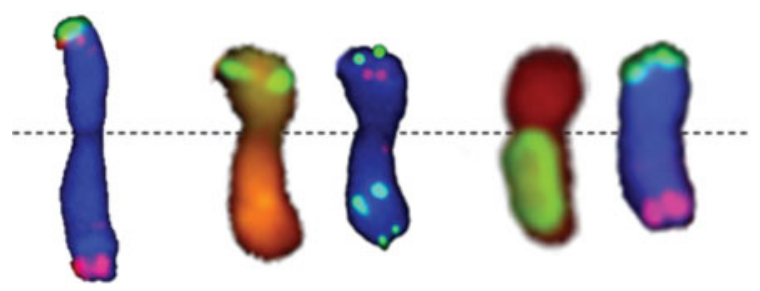

Figure 9 The pattern of dual-color FISH of 7V chromosome and two translocation chromosomes, from left to right, chromosome $7 \mathrm{~V}$, short arm translocation of chromosome 7V (in TV54-5-5-22) and long arm translocation of chromosome 7V (in TV54-5-5-21), the pSc119.2 is green and pAs1 is red.

morphisms.

FISH has been developed for the physical mapping of repeated DNA sequences on chromosomes and genomes. In the tribe Triticeae, localized tandem repeated sequences are useful as cytogenetic markers for chromosome identification [25-29] and can be applied for the characterization of additions, substitutions and translocations [30-32].

The distributions of repeated sequences pSc119.2 and pAs1 in wheat and its related species have been described widely. In hexaploid wheat, the repeated sequence pSc119.2 is mainly located on the B genome, while pAs1 is mainly located on the D genome [33]. However, both of these repeated sequences are distributed on all the chromosomes (1R-7R) of S. cereale [34]. In our study, it was found that the repeated sequences, $\mathrm{pSc} 119.2$ and $\mathrm{pAs} 1$, were also distinctly distributed on all the individual chromosomes (1V-7V) of $H$. villosa. For example, pSc119.2 was mainly

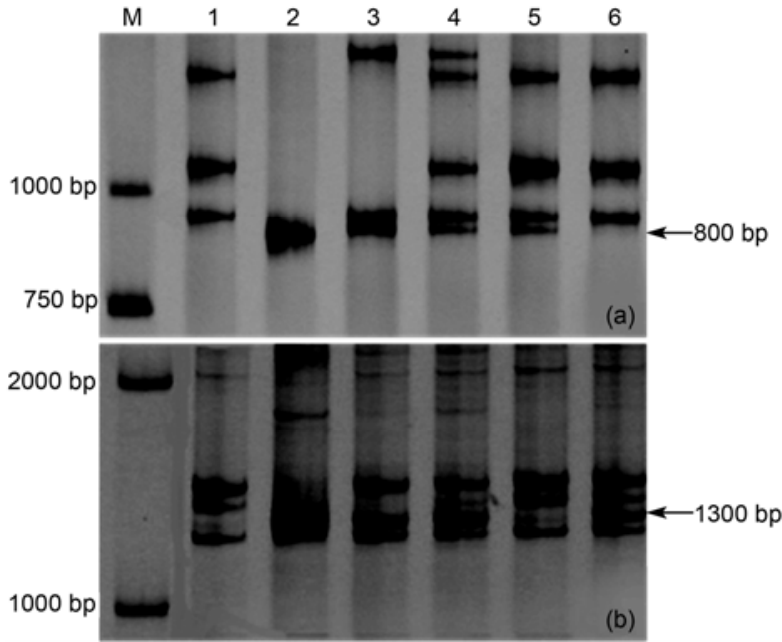

Figure 10 An example of PCR analysis of the two translocation chromosomes using markers $\mathrm{X}_{\mathrm{CINAU} 256-800}(7 \mathrm{VS})$ and $\mathrm{X}_{\mathrm{CINAU} 261-1300}$ (7VL). (a) Amplification of primer CINAU256, (b) amplification of primer CINAU261, arrows show a specific band for chromosome 7V. M, DNA Marker DL2000; 1, Chinese Spring; 2, H. villosa; 3, T. durum-H. villosa amphiploid; 4, DA7V; 5, TV54-5-5-22; 6, TV54-5-5-21.

distributed at the terminal ends of all chromosomes from $1 \mathrm{~V}$ to $7 \mathrm{~V}$, while pAs1 was located not only on the terminal regions, but also on the interstitial regions of all the $H$. villosa chromosomes. The similar distribution of both repetitive sequences in the $\mathrm{V}$ and $\mathrm{R}$ genomes implied that the relationship between the $\mathrm{V}$ and $\mathrm{R}$ genomes may be much closer than that between the $\mathrm{V}$ genome and $\mathrm{A}, \mathrm{B}$ or $\mathrm{D}$ genomes.

Satellite chromosomes of alien species could be easily detected by carmine staining owing to the obvious cytogenetic marker, secondary constriction. However, when the satellite chromosomes of alien species, such as $1 \mathrm{~V}$ of $H$. villos $a$ and $1 \mathrm{R}$ of $S$. cereale, were transferred to the wheat background, the secondary constriction of the satellite chromosomes could not be expressed and detected by carmine staining. In this study, 45S rDNA not only appeared on the short arm of satellite chromosome $1 \mathrm{~V}$ in the $H$. villosa background, but it also appeared in the 1VS whose secondary constriction was hidden in the wheat background (Figure 4). Therefore, for the identification of alien satellite chromosomes in the wheat background, the molecular-cytogenetic marker, $45 \mathrm{~S}$ rDNA, is a good choice.

This study also used the repeated sequence, $5 \mathrm{~S}$ rDNA. It was found that this signal could be observed only on chromosome 5VS in H. villosa, and the signal could be stably detected in the common wheat background (Figure 4). Elite genes that control the grain end-product quality, such as endosperm storage proteins and grain hardness, have been located on 1VS and 5VS [6,35], but these good genes have not been used in commercial wheat varieties. To transfer this quality related genes from $H$. villosa to common wheat, the development of chromosome translocations that involve $1 \mathrm{~V}$ and $5 \mathrm{~V}$ should be effective. In such chromosome engi- 
neering processes, the precise identification of the alien chromosomes in the wheat background is a critical step, and the distributed pattern of $45 \mathrm{~S}$ rDNA and $5 \mathrm{~S}$ rDNA in the 1VS and 5VS, as revealed in this study, will be very helpful.

The combination of GISH and FISH assays using a set of wheat- $H$. villosa disomic addition lines could provide a unique strategy for assigning the FISH signals of repetitive sequences to individual chromosomes in order to yield a $H$. villosa molecular karyotype (Figure 3 ). As a demonstration of the utility of these FISH landmarks, two telocentric chromosomes and two whole-arm translocation chromosomes were identified with the use of pSc119.2 and pAs1 as probes. The result of this determination was confirmed with the simultaneous use of chromosome arm-specific molecular markers. Currently, FISH landmarks combined with molecular marker analysis strategies is being used to identify various translocation lines with different breakpoints that cover the entire genome of $H$. villosa. It appears that this is a highly efficient and high-throughput approach that should promote progress in alien chromosome manipulation, gene mapping and utilization.

This work was supported by the National Natural Science Foundation of China (30871519), the National Basic Research Program of China (2009CB118304) and the Priority Academic Program Development of Jiangsu Higher Education Institutions.

1 Hyde B B. Addition of individual Haynaldia villosa chromosomes to hexaploid wheat. Am J Bot, 1953, 40: 174-182

2 Chen P D, Liu D J. Cytogenetic studies of hybrid progenies between Triticum aestivum and Haynaldia villosa (in Chinese). J Nanjing Agric Coll, 1982, 5: 1-16

3 Chen Q, Conner R L, Li H, et al. Expression of resistance to stripe rust, powdery mildew and the wheat curl mite in Triticum aestivumHaynaldia villosa lines. Can J Plant Sci, 2002, 82: 451-456

4 Murray T D, De la Pena R C, Yildirim A, et al. A new source of resistance to Pseudocercosporella herpotrichoides cause of eyespot disease of wheat located on chromosome 4V of Dasypyrum villosum. Plant Breed, 1994, 113: 281-286

5 Zhang Q P, Li Q, Wang X E, et al. Development and characterization of a Triticum aestivum-Haynaldia villosa translocation line T4VS4DL conferring resistance to wheat spindle streak mosaic virus. Euphytica, 2005, 145: 317-320

6 Blanco A, Resta P, Simeone R, et al. Chromosomal location of seed storage protein genes in the genome of Dasypyrum villosum (L.) Candargy. Theor Appl Genet, 1991, 82: 358-362

7 De Pace C, Snidaro D, Ciaffi M, et al. Introgression of Dasypyrum villosum chromatin into common wheat improves grain protein quality. Euphytica, 2001, 117: 67-75

8 Chen P D, Liu D J. Identification of Haynaldia villosa chromosomes in alien wheat addition. In: Li Z S, Swaminathan M S, eds. Proceedings of the International Symposium on Chromosome Engineering in Plants. Xi' an, China, 1986. 31-33

9 Liu D J, Chen P D, Wu P L, et al. Triticum durum-Haynaldia villosa amphidiploid (in Chinese). Acta Agron Sin, 1986, 12: 155-162

10 Chen P D, Qi L L, Zhou B, et al. Development and molecular cytogenetic analysis of wheat-Haynaldia villosa $6 \mathrm{VS} / 6 \mathrm{AL}$ translocation lines specifying resistance to powdery mildew. Theor Appl Genet, 1995, 91: 1125-1128

11 Friebe B, Cermeno M C, Zeller F I. C-banding polymorphism and the analysis of nuclear activity in Dasypyrum villosum (L.) Candargy, its added chromosomes to hexaploid wheat and the amphiploid Triticum dicoccum-D. villosum. Theor Appl Genet, 1987, 73: 337-342

12 Dong F G, Chen P D, Liu D J. An improved C-banding technique for Haynaldia villosa chromosomes (in Chinese). Acta Genet Sin, 1991, 18: 525-528

13 Liu D J, Chen P D. N-banding in Haynaldia villosa and Triticum durum-H. villosa Amphidiploid (in Chinese). Acta Genet Sin, 1984, 11: 106-108

14 Gupta P K, Fedak G, Molnar S J, et al. Distribution of a Secale cereale sequence among 25 Hordeum species. Genome, 1989, 32: 383388

15 Rayburn A L, Gill B S. Use of biotin-labeled probes to map specific DNA sequences on wheat chromosomes. J Hered, 1985, 76: 78-81

16 Vershinin A V, Svitashev S K, Gummesson P O, et al. Characterization of a family of tandemly repeated DNA sequences in Triticeae. Theor Appl Genet, 1994, 89: 217-225

17 Gill B S, Friebe B, Endo T R. Standard karyotype and nomenclature system for description of chromosome bands and structural aberrations in wheat (Triticum aestivum). Genome, 1991, 34: 830-839

18 Zhang P, Li W L, Friebe B, et al. Simultaneous painting of three genomes in hexploid wheat by BAC-FISH. Genome, 2004, 47: 979987

19 Sharp P J, Kreis M, Shewry P. Location of $\beta$-amylase sequence in wheat and its relatives. Theor Appl Genet, 1988, 75: 289-290

20 Yuan W Y, Tomita M, Sun S C, et al. Multicolor fluorescence in situ hybridization of the rRNA genes in wheat relatives, Dasypyrum villosum and Thinopyrum intermedium. Bull Fac of Agric, Tottori Univ, 2000, 53: 7-12

21 Bie T D, Cao Y P, Chen P D. Mass production of intergeneric chromosomal translocatons through pollen irradiation of Triticum durumHaynaldia villosa amphiploid. J Integr Plant Biol, 2007, 49: 16191626

22 Cao Y P, Bie T D, Wang X E, et al. Induction and transmission of wheat-Haynaldia villosa chromosomal translocations. J Genet Genomics, 2009, 36: 313-320

23 Flavell R B. Repetitive DNA and chromosome evolution in plants. Philos Trans R Soc Lond B Biol Sci, 1986, 312: 227-242

24 Jiang J, Nasuda S, Dong F, et al. A conserved repetitive DNA element located in the centromeres of cereal chromosomes. Proc Natl Acad Sci USA, 1996, 93: 14210-14213

25 Rayburn A L, Gill B S. Molecular identification of the D-genome chromosomes of wheat. J Hered, 1986b, 77: 253-255

26 Mukai Y, Friebe B, Gill B S. Comparison of C-banding patterns and in situ hybridization sites using highly repetitive and total genomic rye DNA probes of 'Imperial' rye chromosomes added to 'Chinese Spring' wheat. Jpn J Genet, 1992, 67: 71-83

27 Anamthawat-Jonsson K, Heslop-Harrison J S. Isolation and characterization of genome-specific DNA sequences in Triticeae species. Mol Gen Genet, 1993, 240: 151-158

28 Zhang P, Li W L, Fellers J, et al. BAC-FISH in wheat identifies chromosome landmarks consisting of different types of transposable elements. Chromosoma, 2004, 112: 288-299

29 Badaeva E D, Zoshchuk S A, Paux E, et al. Fat element-a new marker for chromosome and genome analysis in the Triticeae. Chromosome Res, 2010, 18: 697-709

30 Yuan W X, Tomita M. Centromeric distribution of 350-family in Dasypyrum villosum and its application to identifying Dasypyrum chromatin in the wheat genome. Hereditas, 2009, 146: 58-66

31 Qi Z J, Du P, Qian B L, et al. Characterization of a wheat-Thinopyrum bessarabicum (T2JS-2BS-2BL) translocation line. Theor Appl Genet, 2010, 121: 589-597

32 Jia J Q, Yang Z J, Li G R, et al. Isolation and chromosomal distribution of a novel Ty1-copia-like sequence from Secale, which enables identification of wheat-Secale africanum introgression lines. J Appl Genet, 2009, 50: 25-28

33 Schneider A, Linc G, Molnár-Láng M. Fluorescence in situ hybridization polymorphism using two repetitive DNA clones in different 
cultivars of wheat. Plant Breed, 2003, 122: 396-400

34 Cuadrado A, Jouve N. Distribution of highly repeated DNA sequences in species of the genus Secale. Genome, 1997, 40: 309-317
35 Zhang R Q, Cao Y P, Wang X E, et al. Development and characterization of a Triticum aestivum-Haynaldia villosa T5VS-5DL translocation line with soft grain texture. J Cereal Sci, 2010, 51: 220-225

Open Access This article is distributed under the terms of the Creative Commons Attribution License which permits any use, distribution, and reproduction in any medium, provided the original author(s) and source are credited.

\section{Supporting Information}

Table S1 Chromosome arm-specific molecular markers for Haynaldia villosa

Figure S1 Amplification of specific primer on DNA of parents and wheat- $H$. villosa addition lines.

Table S2 Specific molecular markers used for the identification chromosome 1V and 5V of H. villosa

Table S3 The result of identification of chromosome specific molecular markers of $1 \mathrm{~V}$ and $5 \mathrm{~V}$

The supporting information is available online at csb.scichina.com and www.springerlink.com. The supporting materials are published as submitted, without typesetting or editing. The responsibility for scientific accuracy and content remains entirely with the authors. 